\title{
Direct and Correlated Effects of Selection on Flight After Exposure to Thermal Stress in Drosophila melanogaster
}

\author{
Robert A. Krebs \\ Cleveland State University, r.krebs@csuohio.edu \\ Kimberly A. Thompson \\ Cleveland State University
}

Follow this and additional works at: https://engagedscholarship.csuohio.edu/scibges_facpub

Part of the Biology Commons

How does access to this work benefit you? Let us know!

Publisher's Statement

The final publication is available at Springer via http://dx.doi.org/10.1007/s10709-005-5704-x

\section{Recommended Citation}

Krebs R and Thompson K. 2006. Direct and correlated effects of selection on flight after exposure to thermal stress in drosophila melanogaster. Genetica 128(1-3):217-25.

This Article is brought to you for free and open access by the Biological, Geological, and Environmental Sciences Department at EngagedScholarship@CSU. It has been accepted for inclusion in Biological, Geological, and Environmental Faculty Publications by an authorized administrator of EngagedScholarship@CSU. For more information, please contact library.es@csuohio.edu. 


\title{
Direct and correlated effects of selection on flight after exposure to thermal stress in Drosophila melanogaster
}

\author{
Robert A. Krebs* \& Kimberly A. Thompson \\ Department of Biological, Geological and Environmental Sciences, Cleveland State University, 2121 Euclid \\ Ave., Cleveland, OH, 44115, USA; *Author for correspondence (Phone: +216-523-7553; Fax: + 216-687- \\ 6972; E-mail: r.krebs@csuohio.edu)
}

Key words: flight, genetic variation, knockdown, natural selection, survival, temperature stress, thermotolerance

\begin{abstract}
To demonstrate how insects may adapt to ecologically relevant levels of heat stress, we performed artificial selection on the ability of Drosophila melanogaster to fly after an exposure to a high but non-lethal thermal stress. Both tolerance and intolerance to heat stress arose very quickly, as only a few generations of selection were necessary to cause significant separation between high and low lines for heat tolerance. Estimates of heritability based on the lines artificially selected for increased flight ability ranged from 0.024 to 0.052 , while estimates of heritability based on the lines selected for the inability to fly after heat stress varied between 0.035 and 0.091 . Reciprocal $F_{1}$ crosses among these lines revealed strong additive effects of one or more autosomes and a weaker X-chromosome effect. This variation apparently affected flight specifically; neither survival to a more extreme stress nor knockdown by high temperature changed between lines selected for high and low heat tolerance as measured by flight ability. As the well-studied heat-shock response is associated with heat tolerance as measured by survival and knockdown, the aspects of the stress physiology that actually affect flight ability remains unknown.
\end{abstract}

\section{Introduction}

Many physiological changes occur as temperature rises (Feder, 1996). High-temperature stress affects organisms in a variety of ways, and therefore the variation underlying this tolerance should depend upon how tolerance to stress is measured (Bennett, 1987; Hoffman et al., 1997; Shine et al., 2000; Sørensen, Dahlgaard \& Loeschcke, 2001). Consequences to fitness after heat stress may progress from a decline in oviposition and fertility, reduction in body size, a failure to mate, the cessation of locomotion (knockdown) and increased mortality (Feder \& Krebs, 1997; Fasolo \& Krebs, 2004).

One of the best-known responses to stress is the heat-shock response, which includes induction of Hsp70 in Drosophila melanogaster, as well as a potential suite of other physiological changes (Feder \& Hofmann, 1999). Stress responses to high temperature can be tissue-specific, both for expression of heat-shock proteins and physical damage, which suggests why different traits are affected at different temperatures (Krebs \& Feder, 1997). Flight musculature in flies tends to produce little if any Hsp70 (Denlinger et al., 1991; Patton \& Krebs, 2001), and therefore other stress responses may be more important for protecting flight and related behaviors (ElWadawi \& Bowler, 1995, 1996). Loss of flight ability has obvious ecologically consequences, and yet the ability to fly imposes known metabolic costs. For example, tradeoffs between investment in flight musculature and fecundity exist in Gryllus firmus (Crnokrak \& Roff, 2002). Complex modifications to metabolism 
can underlie these changes, in which hormones tightly regulate genetically based differences (Zera \& Zhoa, 2004; Zera, 2005). Furthermore, genetic variation in flight performance exists even under standard laboratory conditions (Curtsinger \& Laurie-Ahlberg, 1981; Montooth, Marden \& Clark, 2003).

Therefore, flight is likely to vary genetically in D. melanogaster after exposure to thermal stress, as found among strains of D. mojavensis (Krebs \& Thompson, 2005), but because those strains had been reared several years in the laboratory, levels of variation may not have been applicable to natural populations. Here we analyzed genetic variation in flight performance after stress within a recently collected population of $D$. melanogaster, with three specific goals in mind: first, we performed artificial selection to produce a pair of non-inbred high- and low-flight lines based on performance after thermal stress; second, we used these lines to test predictions that flight ability after heat stress was related to other measures of heat stress resistance, i.e. survival (e.g., Morrison \& Milkman, 1978; Loeschcke, Krebs \& Barker, 1994) and knockdown resistance after heat shock (Huey et al., 1992); and third, we assessed genetic differences between lines using $F_{1}$ reciprocal crosses in a preliminary genetic assessment of differences in flight ability of our selected lines.

\section{Methods}

Ten isofemale lines of $D$. melanogaster were used in this experiment that originated from the desert region of San Blas, Nayarit, Mexico in January 2004 (Tucson Drosophila species stock center, ID \#14021-0231.26). We obtained these lines in summer, 2004, which meant these lines had been in laboratory culture no more than 5-6 generations before the selection experiments began. Similar numbers of adults from each line were pooled as virgins and reared on a medium of cornmeal, molasses, agar, tegosept, and proprionic acid in an environmental chamber kept at a constant temperature of $25^{\circ} \mathrm{C}$ for three generations to produce 10 bottle cultures of a large, inter-crossed population prior to artificial selection.

After three generations, all bottles were cleared in the morning and then 231 males and $204 \mathrm{fe}$ males were collected under $\mathrm{CO}_{2}$ anesthesia, and placed into cotton-topped vials containing culture medium. Ten to 12 males and females were placed in each vial. Five days later, all 435 flies were heat shocked in a water bath at $37 \pm 0.1^{\circ} \mathrm{C}$ for $60 \mathrm{~min}$ (model 7305 from Polyscience, Niles, IL). Although in nature, flies would heat up more slowly than when moved directly to $37^{\circ} \mathrm{C}$, this treatment has proved useful for inducing stress responses (Hoffmann \& Watson, 1993; Huey \& Kingsolver, 1993). In preparation for immersion, the vials of flies were inverted with a damp rubber stopper placed over the cotton to maintain high internal humidity. Once lifted from the baths, rubber stoppers were removed from each vial and the flies were allowed to recover for $60 \mathrm{~min}$ at $25^{\circ} \mathrm{C}$ before assaying for flight ability.

The selection threshold criterion used for establishing two high flight selection lines was sustained flight for no less than a distance of $12 \mathrm{~cm}$, a condition met by 98 females and 77 males. The criterion for the two low lines required no visible wing damage, the ability to walk, but little ability to fly or to hover. To differentiate between an unwillingness to fly and an inability to fly, individuals were prodded with a camel hair paintbrush, a technique that is commonly used to induce walking when more severe stress treatments are applied (Loeschcke, Krebs \& Barker, 1994). Only when flight could not be induced were flies scored as lacking flight ability. Ninety-nine females and 127 males met those conditions. High-flight and low-flight lines were then produced by pooling approximately 10 males and 10 females in each bottle and randomly assigning bottles as either high/low line 1 or high/low line 2 depending whether flight capable or non-flying individuals were combined.

In each subsequent generation, $12-15$ vials of males and females (10-12 flies per vial) were collected for continued selection in each of the two high-flight lines and the two low-flight lines (about 150 males and 150 females for each line, each generation). Only individuals that flew were saved in the high-flight lines, and only those that failed to fly were saved for the low-flight lines. Each line was then reconstructed from all selected flies and reared in five fresh bottles of medium. Selection was performed on 4- to 5-day-old flies. To maintain high population numbers in each line, all adults had 3 days to recover from the heat treatment before mixing them for rearing. Adults 
oviposited in bottles for 5 days after which they were discarded to avoid over-crowding, although no attempt was made to explicitly control larval densities. Collection of adults began only after substantial numbers of flies began to emerge from bottles; emerging adults were cleared, discarded, and newly emerged virgins were collected less than $12 \mathrm{~h}$ later.

To recapture flies after flight selection, a chamber was constructed of Plexiglas $(50 \times 50 \times 40 \mathrm{~cm})$, which had netting with arm slits on both sides for access. This chamber allowed release of heat-shocked flies and recapture of individuals that flew with a standard glass tube aspirator. No anesthesia was required and $95 \%$ of all individuals that flew were recaptured, returned to clean food vials, and used to establish the next generation of high lines. Low line individuals were more easily assayed and recaptured using a simple white background on an open tabletop.

After four generations of selection when high and low-flight lines had significantly diverged, reciprocal cross progeny were produced among all four lines (the two high-flight and two low-flight lines). Virgin males and females of each strain were collected and used either to start pure strain cultures or for reciprocal $F_{1}$ crosses between these lines, resulting in 16 sets of crosses: four of each selected strain, and two reciprocal sets of each of the six possible crosses between strains. Two bottles of each cross were prepared, and these adults were transferred to fresh medium after 3 and 6 days to ensure sufficient progeny for analysis. As new flies emerged, they were collected within $12 \mathrm{~h}$, and 10-12 males or females were placed in each glass vial. Four-to-five-day-old flies were used as in the selection protocol, and flies were heat treated at $37 \pm 0.1{ }^{\circ} \mathrm{C}$ for $60 \mathrm{~min}$, and given $60 \mathrm{~min}$ to recover before being assayed for flight. Ten replicates for each cross were collected over a 7-day-period, and one vial of males and one of females per cross were heat treated together. Thus, the experiment and analyses of variance were completely balanced.

After assaying reciprocal cross progeny, the adults emerging from the parental strains were tested for survival and knockdown as a response to a more extreme stress. For both of these more commonly applied tests of thermotolerance, adults were collected as above. Survival was tested one generation after completing tests of reciprocal crosses, and knockdown time was assayed for the following generation. Thus, selection had been relaxed for two generations prior to initiating these experiments.

For the test of survival, three blocks of flies were collected; each block had five vials of males and five of females per selection line, and each vial contained 15-20 flies. These blocks of flies were exposed to slightly different heat stresses: block 1 was treated at $38 \pm 0.1^{\circ} \mathrm{C}$ for $60 \mathrm{~min}$, block 2 received $1 \mathrm{~h}$ at $38.5 \pm 0.1^{\circ} \mathrm{C}$ for $60 \mathrm{~min}$, and block 3 received $1 \mathrm{~h}$ at $38.3 \pm 0.1^{\circ} \mathrm{C}$ for $60 \mathrm{~min}$. These temperature adjustments were made to produce mean survival values in the range of $0.3-0.7$, insuring that the variance among lines would be similar even if their means varied significantly. Survival was scored as the ability for a fly to walk $24 \mathrm{~h}$ later.

Knockdown time was scored on individual flies, using 20 males and females from each line. A 4- to 5-day-old adult was placed in a glass vial and immersed at $39^{\circ} \mathrm{C}$. This fly was observed until it fell and could no longer right itself, and the time for this event was recorded to the nearest second. A temperature of $39^{\circ} \mathrm{C}$ was chosen in order to knockdown a majority of flies within $20 \mathrm{~min}$ (range $=5-22 \mathrm{~min}$ ), enabling analysis of 160 flies in the same generation.

Analyses of line variation and correlated effects were performed in SAS (1998). Even though two replicates were established for each selection treatment, the effect of selection was tested in a nested design, using variation between lines within selection treatment in the denominator. For the analysis of genetic differences, each line was treated as an independent entity. This procedure restricts hypotheses testing to the specific lines under study, but enables comparison of underlying variation and associative effects related to these differences.

To quantify genetic variation, estimates of the selection intensity, which is the selection differential $(S)$ in standard deviation units, and the estimate of heritability (where the selection response, $R=h^{2} / S$ ) were calculated for each generation (Falconer, 1981, pp. 172-174). Tests of significance from zero were based on whether confidence limits, determined from the standard errors, overlapped zero. But, these estimates may be biased because they were based on truncation selection on a threshold trait. This procedure may overestimate 
the selection differential, as all parents for the subsequent generation had phenotypic values of 0 (cannot fly) or 1 (able to fly). Variation in each parent generation was estimated from replicate vial means using 10-12 flies per vial to convert the binomial measurement into a quantitative one (Krebs \& Loeschcke, 1997).

\section{Results}

Through four generations of selection, the two high lines and two low lines diverged significantly in the proportion of individuals that could fly after exposure to thermal stress $\left(F_{1,2}=26.1, p<0.05\right)$. Regression coefficients for males and females of each strain are presented in Table 1, with both low lines declining significantly in the proportion that flew while flight frequency increased significantly in both high lines. Initially, $48 \%$ of all females and $33 \%$ of all males flew after an exposure to a $1-\mathrm{h}$ $37^{\circ} \mathrm{C}$ stress. Selection in the high lines increased flight ability after thermal stress to about $90 \%$ in females and to $80 \%$ in males (Figure 1). In contrast, flight ability in low lines decreased to $20 \%$ in females and $10 \%$ in males. Females flew at a higher frequency than males in all lines $\left(F_{1,441}=195, p<0.001\right)$.

Because flight is a threshold trait, all parents satisfied the criteria of no flight after stress (low lines) or flight after stress (high lines). Therefore the initial intensity of selection is very large, which caused rapid selection despite low, but significant, estimates of heritability (Table 2).
Selection intensity (and the associated response to selection) declined across generations in the low lines that rapidly lost the ability to fly, but remained large in the high-flight lines predominantly due to reduced estimates of variance in flight. The outcome of this difference in artificial selection to increase and decrease flight ability was that the two high lines continued to increase in flight performance while the selection response in the two low lines began to plateau by the fourth generation of selection (Figure 1). Variation among the progeny of reciprocal crosses (Figure 2) was explained predominantly by strong effects of male parent $\left(F_{3,318}=63.0, p<0.001\right)$, female parent $\left(F_{3,318}=112.5, p<0.001\right)$, and gender of the flies tested $\left(F_{1,318}=82.4, p<0.001\right)$. Basically, hybrids among the different strains, especially high and low lines, tended to be intermediate, while gender differences persisted. None of the interaction effects were significant, although an effect of male strain was marginal $(p<0.06)$.

Two specific contrasts suggested that genetic differences in flight ability among our selection lines were largely additive. Hybrid male offspring whose female parent was from high-line 2 (the line with the highest tolerance to heat, see Figure 1), consistently flew more often than did male progeny of the reciprocal cross, where paternal parent originated from high-line 2. Also, hybrids between the two low lines had higher flight ability after heat stress than did offspring possessing parental genes from only one of these low lines (Figure 2), except for male offspring of L1 mothers.

Table 1. Regression coefficients of flight frequency for males and females from two lines of D. melanogaster that were selected for their ability to fly after exposure to thermal stress and two lines selected for a failure to fly

\begin{tabular}{lllllll}
\hline Line & Gender & $N$ & Intercept & Slope & $r^{2}$ & $F$ value \\
\hline Low 1 & Females & 56 & $0.44 \pm 0.05$ & $-0.098 \pm 0.019$ & 0.33 & $5.3^{* * *}$ \\
Low 1 & Males & 56 & $0.24 \pm 0.03$ & $-0.057 \pm 0.011$ & 0.31 & $5.1^{* * *}$ \\
Low 2 & Females & 55 & $0.38 \pm 0.05$ & $-0.046 \pm 0.019$ & 0.10 & $2.5^{*}$ \\
Low 2 & Males & 53 & $0.29 \pm 0.04$ & $-0.055 \pm 0.015$ & 0.22 & $3.8^{* * *}$ \\
High 1 & Females & 55 & $0.57 \pm 0.04$ & $0.084 \pm 0.013$ & 0.43 & $6.4^{* * *}$ \\
High 1 & Males & 57 & $0.46 \pm 0.04$ & $0.069 \pm 0.015$ & 0.26 & $4.5^{* * *}$ \\
High 2 & Females & 55 & $0.53 \pm 0.05$ & $0.087 \pm 0.017$ & 0.32 & $5.1^{* * *}$ \\
High 2 & Males & 55 & $0.32 \pm 0.04$ & $0.129 \pm 0.016$ & 0.56 & $8.3^{* * *}$ \\
\hline
\end{tabular}

${ }^{*} p<0.05 ; * * * p<0.001$.

Slope quantifies change in flight ability per generation with $r^{2}$, the proportion of the variance in this response explained by the slope. Input data were frequencies of 10-12 individuals per replicate vial $(N)$ scored through four generations of selection. 


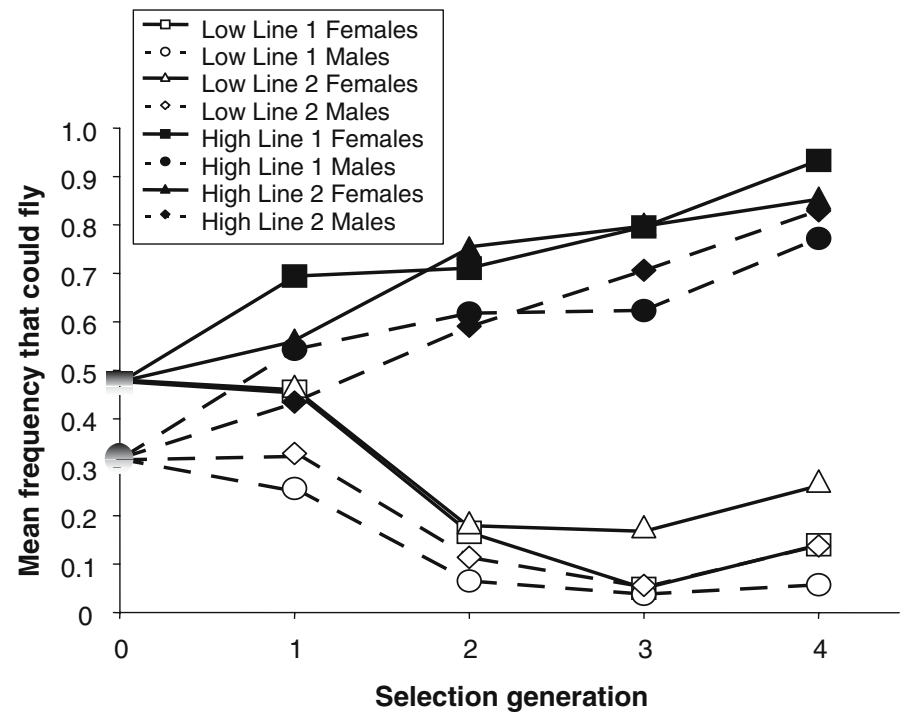

Figure 1. The frequency of flight in males (dotted) and females (solid) in D. melanogaster from two lines selected across four generations for flight capability after exposure to a thermal stress and two other lines selected for an inability to fly after a stress exposure. Each point is estimated from approximately 150 individuals assayed in groups of 10-12.

Table 2. Estimates of the standardized selection differential and heritability for lines $(+1 \mathrm{SE})$ selected either to decrease or to increase flight after exposure to thermal stress

\begin{tabular}{llr}
\hline Generation & Selection intensity & Estimate of $h^{2}$ \\
\hline Low-flight lines & & \\
1 & $4.48 \pm 0.93$ & $0.035 \pm 0.012^{*}$ \\
2 & $3.88 \pm 0.33$ & $0.091 \pm 0.014^{*}$ \\
3 & $1.98 \pm 0.30$ & $0.038 \pm 0.013^{*}$ \\
4 & $0.83 \pm 0.23$ & $<0$ \\
High-flight lines & & \\
1 & $3.84 \pm 0.42$ & $0.034 \pm 0.012^{*}$ \\
2 & $3.57 \pm 0.28$ & $0.034 \pm 0.012^{*}$ \\
3 & $2.74 \pm 0.32$ & $0.024 \pm 0.008^{*}$ \\
4 & $3.17 \pm 0.42$ & $0.052 \pm 0.006^{*}$ \\
\hline
\end{tabular}

$* p<0.05$.

Each estimate was computed separately by sex for each line, and then averaged across the selection treatments. Standard errors apply to variation among these four measurements.

Selection for high- or low-flight ability after heat stress had no significant influence on survival after exposure to a higher temperature stress or direct knockdown from exposure to high temperatures (Figure 3). However, more high-flight ability flies survived the high temperature stress treatment. The only significant effects involved gender where females survived heat stress better than males $(p<0.001)$. By contrast, males remained upright, avoiding knockdown at $39^{\circ} \mathrm{C}$ longer than females $(p<0.01)$. Therefore, neither of these traits showed a significant correlation with temperature tolerance as measured by flight ability.

\section{Discussion}

Flight ability after exposure to high temperature responds rapidly to selection, as has been observed for other tests of stress in Drosophila (Huey et al., 1992; McColl, Hoffmann \& McKechnie, 1996; Gilchrist \& Huey, 1999; Bubliy \& Loeschcke, 2005). Both high lines responded to selection for increased flight ability after thermal stress reaching $80-90 \%$ of flies that flew in each line. In the low lines, flight ability declined rapidly such that only $10-20 \%$ of flies flew after exposure to heat stress, even though estimates of heritability ranged between only 0.02 and 0.09 .

Patterns of genetic variation between the high and low lines suggest a strong additive effect. Between lines, only a small X-chromosome effect separated the two high lines while a hybrid vigor may affect offspring between the two low lines. The possibility that deleterious traits reducing flight ability exist was suggested previously based 


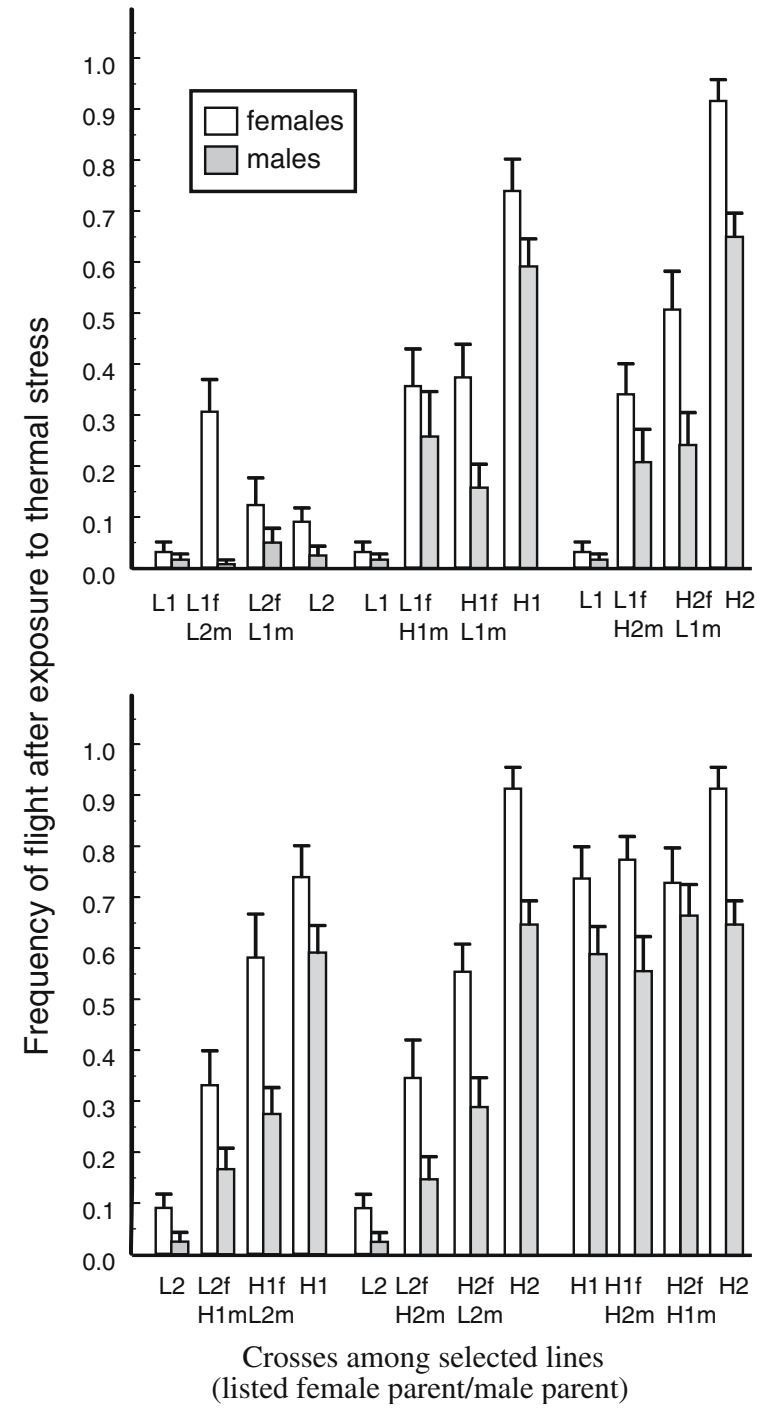

Figure 2. The frequency of flight $( \pm 1 \mathrm{SE})$ in males (shaded) and females (clear) from all reciprocal crosses among the four lines selected either for flight or for an inability to fly after exposure to thermal stress. Pure-strain results are repeated at each location, as indicated.

on strong hybrid vigor in crosses among laboratory strains of D. mojavensis (Fasolo \& Krebs, 2004; Krebs \& Thompson, 2005). Overall, these results show the potential for a small array of genes to contribute to genetic variation in natural populations with respect to flight ability after exposure to heat stress.

There are several implications to the selection responses observed here. At least some natural populations of $D$. melanogaster have the ability to respond rapidly to heat stress allowing for flight
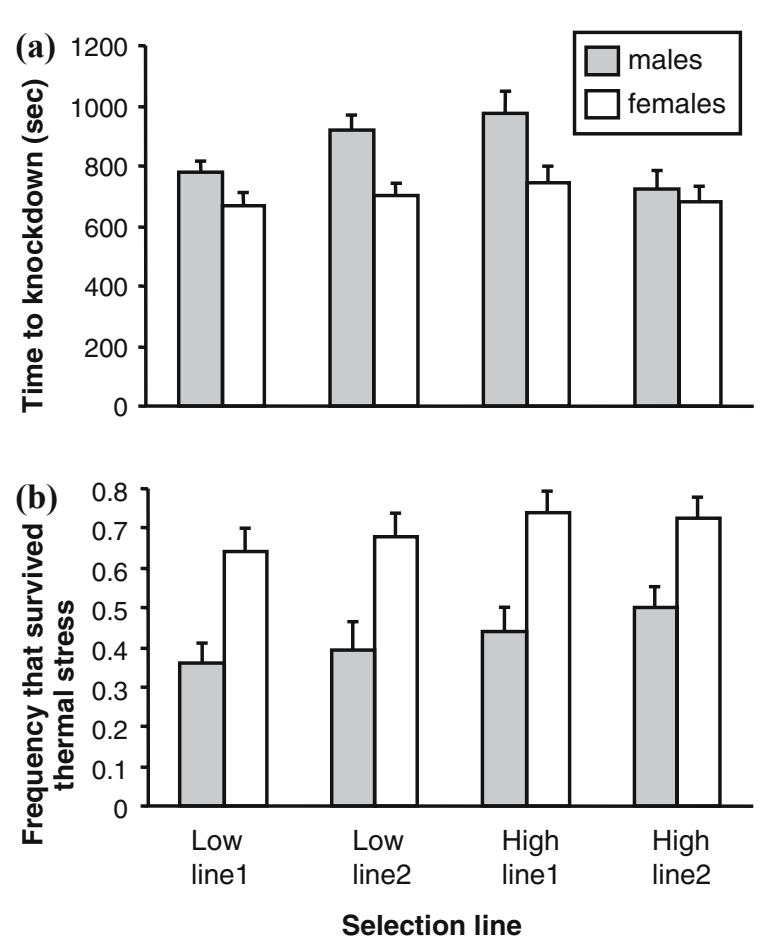

Figure 3. For each of the selected lines, (a) the mean time required for a $39^{\circ} \mathrm{C}$ heat stress to knockdown flies $( \pm 1 \mathrm{SE})$ and (b) the mean frequency of flies from each line that survived a heat stress of about $38.3^{\circ} \mathrm{C}$ for $1 \mathrm{~h}( \pm 1 \mathrm{SE})$.

ability under environmental extremes. Unlike our earlier reports, the variation cannot be attributed simply to deleterious traits present in the population (Krebs \& Thompson, 2005), as the lines rapidly responded to selection to both increased and decreased flight ability. These are the types of results that have been found previously when selection has been focused on survival tolerance to heat (e.g., Morrison \& Milkman, 1978; Krebs \& Loeschcke, 1996; Lansing, Justesen \& Loeschcke, 2000). An important difference here is that selection was possible at much lower temperatures than in experiments assessing survival. This stress level does not affect reproductive ability of the flies (David et al., 2005), and therefore selection could be easily imposed in sequential generations, although the affected underlying cause of variation likely differs (Berrigan, 2000).

Another outcome of selection was that direct effects on flight rapidly changed without any coincident effects on survival or knockdown. Bubliy and Loeschcke (2005) also found low levels of concordance among line differences when 
comparing a select group of stress resistance measures that included stress effects of survival from cold or heat, desiccation, starvation and heat-knockdown time. One concern in any of these analyses is that all measures of resistance cannot be measured concurrently in the same population, and therefore we scored knockdown and survival 1-2 generations after completing selection on flight. However, Krebs and Loeschcke (1997) found that natural variation in survival of $D$. buzzatii after stress persists over several generations, and lines of $D$. melanogaster reared in the absence of stress in the laboratory remain very consistent in stress tolerance as measured by survival even over periods of years (Krebs et al., 2002). Therefore, these low trait correlations are unlikely to be a consequence of relaxed selection from rearing under benign conditions.

Because the genetic basis of different measures of stress resistance seems to vary, what are the possible underlying loci for these traits? Within assays of survival and also knockdown, variation in the expression of $\mathrm{Hsp} 70$ or allelic variation in genes for this protein often have been implicated (Hoffmann, Loeschcke \& Sørensen, 2003), because some $h s p 70$ alleles, as well as knockdown resistance from heat, can be observed to follow a latitudinal cline (Bettencourt et al., 2002; Hoffmann, Anderson \& Hallas, 2002). Higher Hsp70 expression likewise aids survival (Feder \& Krebs, 1997; Feder \& Hofmann, 1999), and selection to increase Hsp70 even improves survival following stress (Feder et al., 2002), although expression of Hsp70 is less in Drosophila flight muscle than in other parts of the fly (Patton \& Krebs, 2001). Selection on knockdown resistance also shows a strong link to other Hsps, as it can alter Hsp68 concentration and that of Hsr Omega (McColl, Hoffmann \& McKechnie, 1996). However, gender effects are reversed between flight resistance and knockdown resistance in the experiments reported here. For these reasons, we do not predict that variation in Hsp70 expression predominantly controls variation in flight after stress.

Thus, what aspects of the physiology actually responded here to selection remains unknown. The ability to fly underlies the evolutionary success of insects, yet, as Luckinbill et al. (2005) highlight, relatively few studies have considered the genetics of the complex processes that allow flight. Direct flight muscle control of wing beat and orientation
(Fry, Sayanman \& Dickinson, 2003), as well as rotation and timing of the wing beats to maintain lift on the upstroke (Ellington et al., 1996; Dickinson, Lehmann \& Sane, 1999), all are likely controlled by numerous developmental pathways. Which of these fail under the effects of heat is unknown. One proposed impact of heat stress is that reduced oxidative respiration in mitochondria can affect flight (ElWadawi \& Bowler, 1996), but that cause leads to the prediction of variation in mitochondrial performance, which would be observed as a maternal effects if present. None was observed.

Thus, variation in flight after stress may be completely unrelated to various known stress responses, and instead be linked to a deficiency in one of the basic physiological processes that enable flight. In their study of genetic variation in flight duration and rate, Luckinbill et al. (2005) also found predominantly additive effects controlling variation among recombinant inbred lines of $D$. melanogaster. While we do not believe that the selection protocol used here could have altered flight performance directly in so few generations, it is suggestive that the thermal environment may also influence some of the factors that underlie natural variation in flight performance. Clearly, these recent findings suggest that both environmental and genetic components of variation in flight performance may be understudied aspects of life history variation.

\section{Acknowledgements}

We thank Owen Lockhart for assistance collecting data, and Cal Borden, Brian Bettencourt, Bill Etges and two anonymous reviewers for comments on the manuscript.

\section{References}

Bennett, A.F., 1987. Interindividual variability: an underutilized resource, pp. 147-165 in New Directions in Ecological Physiology edited by M.E. Feder, A.F. Bennett, W.W. Burggren \& R.B. Huey. Cambridge University Press, Cambridge, MA.

Berrigan, D., 2000. Correlations between measures of thermal stress resistance within and between species. Oikos 89: 301304. 
Bettencourt, B.R., I.Y. Kim, A.A. Hoffmann \& M.E. Feder, 2002. Response to natural and laboratory selection at the Drosophila hsp70 gene. Evolution 56: 1796-1801.

Bubliy, O.A. \& V. Loeschcke, 2005. Correlated responses to selection for stress resistance and longevity in a laboratory population of Drosophila melanogaster. J. Evol. Biol. 18: 789-803.

Crnokrak, P. \& D.A. Roff, 2002. Trade-offs to flight capability in Gryllus firmus: the influence of whole organism respiration rate on fitness. J. Evol. Biol. 15: 388-398.

Curtsinger, J.W. \& C.C. Laurie-Ahlberg, 1981. Genetic variability and flight metabolism in Drosophila melanogaster I. Characterization of power output during tethered flight. Genetics 98: 549-564.

David, J.R., L.O. Araripe, M. Chakir, H. Legout, B. Lemos, G. Petavy, C. Rohmer, D. Joly \& B. Moreteau, 2005. Male sterility at extreme temperatures: a significant but neglected phenomenon for understanding Drosophila climatic adaptations. J. Evol. Biol. 18: 838-846.

Denlinger, D.L., K.H. Joplin, C.-P. Chen \& R.E. Lee Jr., 1991. Cold and heat shock, pp. 131-148 in Insects at Low Temperature, edited by R.E. Lee Jr. \& D.L. Denlinger. Chapman and Hall N. Y.

Dickinson M.H., Lehmann F.-O., Sane S.P., 1999. Wing rotation and the aerodynamic basis of insect flight. Science 284: 1954-1960.

Ellington, C.P., C. van den Berg, A.P. Willmott \& A.L.R. Thomas, 1996. Leading edge vortices in insect flight. Nature 384: 626-630.

ElWadawi, R. \& K. Bowler, 1995. The development of thermotolerance protects blowfly flight-muscle mitochondrial function from heat damage. J. Exp. Biol. 198: 24132421.

ElWadawi, R. \& K. Bowler, 1996. The effect of in vivo heat treatments on blowfly flight muscle mitochondrial function: effects on partial reactions of the respiratory chain. J. Therm. Biol. 21: 403-408.

Falconer, D.S., 1981. Introduction to Quantitative Genetics, 2nd edn. Longman, New York, $340 \mathrm{pp}$.

Fasolo, A.G. \& R.A. Krebs, 2004. Behavioural variation in Drosophila during exposure to thermal stress. Biol. J. Linn. Soc. 83: 197-205.

Feder, M.E., 1996. Ecological and evolutionary physiology of stress proteins and the stress response: the Drosophila melanogaster model, pp. 79-102 in Phenotypic and Evolutionary Adaptation to Temperature edited by I.A. Johnston \& A.F. Bennett. Cambridge University Press, Cambridge, MA.

Feder, M.E. \& G.E. Hofmann, 1999. Heat-shock proteins, molecular chaperones, and the stress response. Annu. Rev. Physiol. 61: 243-282.

Feder, M.E. \& R.A. Krebs, 1997. Natural and genetic engineering of the heat-shock protein Hsp70 in Drosophila melanogaster: consequences for thermotolerance. Amer. Zool. 38: 503-517.

Feder, M.E., T.B.C. Bedford, D.R. Albright \& P. Michalak, 2002. Evolvability of Hsp70 expression under artificial selection for inducible thermotolerance in independent populations of Drosophila melanogaster. Physiol. Biochem. Zool. 75: 325-334.
Fry, S.N., R. Sayaman \& M.H. Dickinson, 2003. The aerodynamics of free flight maneuvers in Drosophila. Science 300: 495-498.

Gilchrist, G.W. \& R.B. Huey, 1999. The direct response of Drosophila melanogaster to selection on knockdown temperature. Heredity 83: 15-29.

Hoffmann, A.A. \& M. Watson, 1993. Geogrpahical extremes in the acclimation responses of Drosophila to temperature extremes. Am. Nat. 142: S93-S113.

Hoffmann, A.A., A. Anderson \& R. Hallas, 2002. Opposing clines for high and low temperature resistance in Drosophila melanogaster. Ecol. Lett. 5: 614-618.

Hoffmann, A.A., V. Loeschcke \& J.G. Sørensen, 2003. Adaptation of Drosophila to temperature extremes: bringing together quantitative and molecular approaches. J. Therm. Biol. 28: 175-216.

Hoffmann, A.A., H. Dagher, M. Hercus \& D. Berrigan, 1997. Comparing different measures of heat resistance in selected lines of Drosophila melanogaster. J. Insect Physiol. 43: 393405.

Huey, R.B. \& J.G. Kingsolver, 1993. Evolution of resistance to high-temperature in ectotherms. Am. Nat. 142: S21-S46.

Huey, R.B., W.D. Crill, J.G. Kingsolver \& K.E. Weber, 1992. A method for rapid measurement of heat or cold resistance of small insects. Funct. Ecol. 6: 489-494.

Krebs, R.A. \& M.E. Feder, 1997. Natural variation in the expression of the heat-shock protein $\mathrm{Hsp} 70$ in a population of Drosophila melanogaster, and its correlation with tolerance of ecologically relevant stress. Evolution 51: 173-179.

Krebs, R.A. \& V. Loeschcke, 1996. Selection for increased resistance and acclimation to thermal stress in Drosophila buzzatii. Genetics 142: 471-479.

Krebs, R.A. \& V. Loeschcke, 1997. Estimating heritability in a threshold trait: heat-shock tolerance in Drosophila buzzatii. Heredity 79: 252-259.

Krebs, R.A. \& K.A. Thompson, 2005. A genetic analysis of variation for the ability to fly after exposure to thermal stress in Drosophila mojavensis. J. Therm. Biol. 30: 335-342.

Krebs, R.A., S.P. Roberts, B.R. Bettencourt \& M.E. Feder, 2002. Changes in thermotolerance and Hsp70 expression with domestication in Drosophila melanogaster. J. Evol. Biol. 14: 75-82.

Lansing, E., J. Justesen \& V. Loeschcke, 2000. Variation in the expression of $\mathrm{Hsp} 70$, the major heat-shock protein, and thermotolerance in larval and adult selection lines of Drosophila melanogaster. J. Therm. Biol. 25: 443-450.

Loeschcke, V., R.A. Krebs \& J.S.F. Barker, 1994. Genetic variation for tolerance and acclimation to high temperature stress in Drosophila buzzatii. Biol. J. Linn. Soc. 52: 83-92.

Luckinbill, L.S., S. Reddy, V. Dudekonda \& J.W. Curtsinger, 2005. Analysis of two components of flight using recombinant inbred lines of Drosophila melanogaster. Genetica 124: 235-245.

McColl, G., A.A. Hoffmann \& S.W. McKechnie, 1996. Response of two heat shock genes to selection for knockdown heat resistance in Drosophila melanogaster. Genetics 143: 1615-1627.

Montooth, K.L., J.H. Marden \& A.G. Clark, 2003. Mapping determinants in energy metabolism, respiration and flight in Drosophila. Genetics 165: 623-635. 
Morrison, W.W. \& R. Milkman, 1978. Modification of heat resistance in Drosophila by selection. Nature 273: 49-50.

Patton, Z.J. \& R.A. Krebs, 2001. The effect of thermal stress on the mating behavior of three Drosophila species. Physiol. Biochem. Zool. 74: 783-788.

SAS Institute, 1998. SAS Stat Users Guide, Cary, N.C.

Shine, R., P.S. Harlow, M.J. Elphick, M.M. Olsson \& R.T. Mason, 2000. Conflicts between courtship and thermoregulation: the thermal ecology of amorous male garter snakes (Thamnophilis sirtalis parietalis, Colubridae). Physiol. Biochem. Zool. 73: 508-513.

Sørensen, J.G., J. Dahlgaard \& V. Loeschcke, 2001. Genetic variation in thermal tolerance among natural populations of Drosophila buzzatii: down regulation of Hsp70 expression and variation in heat stress resistance. Funct. Ecol. 15: 289-296.

Zera, A.J., 2005. Intermediary metabolism and life history trade-offs: lipid metabolism in lines of the wing-polymorphic cricket, Gryllus firmus, selected for flight capability vs. early age reproduction. Integr. Compar. Biol. 45: 511-524.

Zera, A.J. \& Z.W. Zhao, 2004. Effect of a juvenile hormone analogue on lipid metabolism in a wing-polymorphic cricket: implications for the endocrine-biochemical bases of life-history trade-offs. Physiol. Biochem. Zool. 77: 255-266. 